\title{
Distribución geográfica y ecológica de la nutria de río neotropical (Lontra longicaudis) en Michoacán, México
}

\author{
Tiberio C. Monterrubio-Rico ${ }^{1}$ y Juan F. Charre-Medellín ${ }^{1 *}$
}

Introduction: Considering the general deterioration of Michoacán rivers, increased poaching, the failure to enforce relevant laws in the last 10 years, and the absence of a detailed assessment of the current status of the river otter (Lontra longicaudus) in the state of Michoacán, our main goal was to collect and analyze all available historical and recent evidence for the occurrence of the river otter in this state. The study focused on three regions for which field recordings were obtained during the period 2005 to 2012 . Our second objective was to analyze the ecological conditions associated with these recent records. This information is especially important because the species is protected in Michoacán, and it is an indicator of the environmental quality of water bodies.

Methodology: Historical records of otters in Michoacán were reviewed to establish their reliability and age. The search included scientific papers, theses, and data bases of scientific collections available on line. The current presence of river otters in the state was further documented by diurnal transects along rivers and streams in which visual records, footprint tracks, feces, burrows, latrines, or skulls were obtained. The records were then projected on maps in order to analyze them in the context of biogeographic regions, watersheds, vegetation-types, climate, cities, and rivers.

Results: The presence of river otters in Michoacán was documented by only 10 historical records (Fig. 1, Table 1). Our field work generated 90 records in 17 localities (Fig. 2, Table 1). The records were found in four watersheds. The type of vegetation that is associated with the largest number of records was the tropical deciduous forest $(n=47,52 \%)$, which is characterized by average temperatures between 21 and $28{ }^{\circ} \mathrm{C}$ and annual rainfall between 700 and $1,300 \mathrm{~mm}$.

Discussion and conclusions: Our results represent the most recent records for L. longicaudus for the state of Michoacán, and perhaps for any other state in central-western Mexico. The observed distribution of the river otter confirms its presence in at least three major biogeographic regions of Michoacán, namely Balsas, Sierra Madre del Sur, and Pacific Coast. All the data obtained during the study associate this species with perennial streams and where permanent pools remain during the dry season (Fig. 1). This area may be an important refuge for this species because a hydrological network is spread over environmental heterogeneity encompassing temperate forests in the Sierra Madre del Sur and the Eje Neovolcanico to tropical deciduous forests along the Balsas River and the Pacific coast. In the future, it will be important to document if this otter occurs in the Zicuirán-Infiernillo Biosphere Reserve which seems to have the potential for connectivity with rivers in the state of Guerrero. This would make the Michoacán otters of considerable importance for conservation programs on a regional level.

Key words: climatic profile, Balsas, Mustelidae, skull, water dog.

Laboratorio de Ecología de Vertebrados Terrestres Prioritarios, Facultad de Biología, Universidad Michoacana de San Nicolás de Hidalgo, Morelia, Michoacán 58194, México. Email: tmonter2001@yahoo.com.mx (TCMR); jfcharre@yahoo. com.mx (JFCM)

* Corresponding author 
La nutria de río neotropical (Lontra longicaudis annectens), es uno de los carnívoros en riesgo del centro occidente de México que a pesar de presentar una distribución histórica y potencial amplia, todavía se desconoce su distribución actual, sus condiciones de conservación, y ecología local a lo largo de los principales ríos de la vertiente del Pacífico incluyendo Michoacán. Para el estado encontramos 10 localidades de registros históricos sobre la presencia de la nutría, que no permiten delimitar su distribución actual para las diferentes regiones del estado debido a su dispersión, poca representatividad regional, e imprecisión. Mediante transectos diurnos, obtuvimos 90 registros de 17 localidades que incluyen evidencia fotográfica, y biológica que confirman la presencia actual de nutria de río (L. longicaudis) en tres regiones y cuatro cuencas hidrológicas del estado de Michoacán, México. Todos los registros están asociados a ríos perennes, y la mayoría de los registros se obtuvo en bosques tropicales caducifolios, seguido de bosques de pino-encino. Se obtuvo el perfil bioclimático para la nutria, considerando temperatura y precipitación. Con base en las distancias y condiciones topográficas que separan los ríos donde se confirmó su presencia entre la Costa Michoacana y la Depresión del Balsas, desconocemos si existe contacto entre las poblaciones de nutria de río de estas regiones. La amplia distribución observada para la nutria en las tres regiones permite asumir que la especie en Michoacán cuenta con una población importante, pero con escasa disponibilidad de superficie protegida.

Palabras clave: Balsas, cráneo, Mustelidae, perfil climático, perro de agua.

La nutría de río neotropical Lontra longicaudis, también conocida en el medio rural de Mesoamérica cómo "perro de agua" (Reid 1997), presenta una amplia distribución continental desde el norte de México hasta la provincia de Buenos Aires en Argentina (Larivière 1999). Tres subespecies han sido reconocidas globalmente, correspondiendo a México la subespecie L. longicaudis annectens (Larivière 1999). En México la nutria de río se distribuye a lo largo de la vertiente del pacifico desde Chihuahua hasta Chiapas, y por la vertiente del golfo de México desde Yucatán hasta Tamaulipas (Gallo 1997). A pesar de su alta especialización piscívora, la nutria consume desde crustáceos y pequeños mamíferos, hasta aves, reptiles, insectos e incluso frutos (Larivière 1999; Quadros y Monteiro-Filho 2000; Gallo et al. 2008, Guerrero-Flores et al. 2013). La nutria es un carnívoro de la familia Mustelidae que en México incluye tres especies de nutrias (Enhydra lutris, Lontra canadensis y Lontra longicaudis), y cuatro mustélidos terrestres (Taxidea taxus, Mustela frenata, Galictis vittata, Eira barbara), además de la reintroducida Mustela nigripes (Ceballos y Oliva 2005).

La nutria de río se asocia principalmente a cuencas de ríos con aguas claras con corriente lenta o relativamente rápida, también en arroyos secundarios y en lagunas de agua dulce y lagunas costeras de Sinaloa, Guerrero, Veracruz y Yucatán (Sánchez et al. 2007). Generalmente se encuentra por debajo de los 1500 msnm, sin embargo en algunas localidades de México ha sido registrada arriba de los 2000 m (Santo-Moreno et al. 2003; Servín et al. 2003), siendo 3,000 m la mayor altitud en la cual se ha observado en Argentina (Eisenberg y Redford 1999; Larivière 1999). 
Aunque existían antecedentes de la presencia de nutria de río en el estado de Michoacán (Leopold 1959; Gallo 1997; Magaña-Cota 2008), solo existe un ejemplar colectado, el cual está depositado en el Museo de Historia Natural Alfredo Dugés en la ciudad de Guanajuato. El ejemplar fue colectado en 1876 en el municipio de Tangancícuaro, Michoacán por el naturalista Alfredo Dugès en el siglo XIX durante su estancia en México (Magaña-Cota 2008). Sin embargo, hasta hoy, no existía ningún estudio publicado basado en registros contemporáneos que confirmaran, delimitaran y proporcionaran un panorama actual a nivel estatal sobre la distribución de la nutria.

En general hasta antes de este estudio, la información disponible sobre la nutria en Michoacán se encontraba dispersa. Los registros existentes en su mayoría antiguos y anecdóticos, incluso presentaban imprecisiones sobre las localidades de ocurrencia que no corresponden a los sitios de colecta u observación (Leopold 1959; Gallo 1997; Magaña-Cota 2008). El registro más antiguo de nutria es el ejemplar colectado por A. Dugès en 1876, posteriormente se encuentra el de Leopold (1959) que documenta un perro de agua disecado y montado en la Colección del Laboratorio de Limnología de Pátzcuaro, sin datos de procedencia y fecha de colecta. Otro registro procedente de San José de la Montaña (Municipio de Coalcomán), en el que se reporta una piel de un ejemplar cazado en las cercanías (Brand 1961). Álvarez et al. (1987) citó la presencia de nutria en las cercanías del río Tupitina. Gallo (1997), proporcionó posteriormente el conjunto de registros más recientes basados en entrevistas y recorridos que reportan nutria para los ríos Coalcomán, Maruata, Huahua y Popoyuta a lo largo de la Costa de Michoacán (Tabla 1).

Ante el deterioro general de los ríos de Michoacán (Israde-Alcántara 2005), el incremento de caza ilegal por la inseguridad durante los últimos 10 años, y ante la falta de un diagnóstico detallado sobre la situación actual de la nutria en el estado de Michoacán, el principal objetivo de este estudio fue reunir y analizar toda la evidencia disponible, histórica y reciente e integrar un diagnóstico enfocado en tres regiones para las que se obtuvo registros de campo durante el periodo 2005-2012. Como segundo objetivo se analizó las condiciones ecológicas asociadas a los sitios de registro a nivel climático. Esta información es particularmente importante ya que la especie se encuentra listada en la norma oficial, y es indicadora de la calidad ambiental de los cuerpos de agua (Soler 2002).

\section{Material}

Michoacán cuenta con una amplia red de cuencas y sistemas fluviales, cuatro sistemas son lacustres, el Lago de Cuitzeo, el Lago de Pátzcuaro, el Lago de Zirahuén, y el Lago de Chapala, y la Cuenca del río Lerma, situados en el altiplano y en el eje Neovolcánico Transversal. Además existen dos amplios sistemas fluviales que fluyen en la zona neotropical del estado, la Cuenca del Balsas Tepalcatepec y el sistema fluvial costero, los cuales no desembocan en lagos (Bocco et al. 1999). Como parte de distintos proyectos de investigación efectuados, se muestreó en ríos de las tres regiones del estado con mayor cobertura de vegetación original, principalmente en la cuenca del bajo Balsas, la Sierra Madre del Sur, y la costa del Pacífico (Fig. 1). La vegetación de las tres regiones que conforman el área de estudio incluye principalmente el bosque tropical caducifolio y el bosque tropical subcaducifolio. Sin embargo en las partes altas, como en el municipio 
de Arteaga (> a $600 \mathrm{~m}$ ), encontramos bosques templados de encino y pino (CharreMedellín 2009). Todas las comunidades vegetales de la región presentan disturbios antropogénicos como pastoreo y la agricultura en diferentes niveles de intensidad, aunque todavía se pueden encontrar fragmentos continuos $\geq 1000$ has de vegetación primaria, especialmente en los municipios de Arteaga y Aquila (INEGI 2009).

Revisión de literatura y base de datos. Se revisaron los registros históricos de nutria para el estado de Michoacán con la finalidad de establecer su confiabilidad y antigüedad.

La búsqueda incluyó artículos científicos, tesis de grado, bases de datos de colecciones científicas, y bases de datos disponibles en línea como GBIF (The Global Biodiversity Information Facility data bases, http://www.gbif.org/), MANIS (Mammal Networked Information System, http://www.manisnet.org/). Además se contó con la base de datos de los mamíferos de Michoacán proporcionada por la Comisión Nacional para el Conocimiento y Uso de la Biodiversidad (CONABIO), la cual está integrada por 9,186 registros de mamíferos provenientes de 10 proyectos de investigación donde destacan los proyectos "P020 Biodiversidad de los Mamíferos de Michoacán", correspondientes al periodo de colecta de 1967 a 1978, con 6537 registros (Álvarez-Solórzano y López-Vidal 1998), el proyecto "T009 Actualización de la base de datos del Atlas Mastozoológico de México con 1269 registros (Ceballos 2002), y el proyecto "P130 Base de datos de mamíferos de México depositados en colecciones de Estados Unidos y Canadá" (LópezWilchis 1998) con 954 registros.

Trabajo de campo. Como resultado de diversos proyectos de investigación realizados en distintas regiones del estado desde el año 2005 a 2012, se documentó la presencia de la nutria de río en el estado mediante transectos diurnos a lo largo de ríos y arroyos, en los cuales se obtuvo registros visuales directos, y rastros de nutria como huellas, excretas, madrigueras, echaderos, letrinas o esqueletos (Fig. 2). Los recorridos tuvieron una extensión aproximada de 4 a $5 \mathrm{~km}$. Como los registros provienen de diferentes proyectos de investigación efectuados en distintos años, además de limitaciones logísticas e inseguridad, el muestreo no se realizó con diseños experimentales balanceados, y varias localidades fueron visitadas solo una ocasión.

Caracterización geográfica y ecológica de la nutria de río en Michoacán. Los registros obtenidos fueron georeferenciados y proyectados en el espacio geográfico con la finalidad de analizarlos por región biogeográfica, cuenca hidrológica, municipios y ríos (Tabla 1). Para la caracterización ecológica de la especie se construyó el perfil bioclimático de la especie, a partir de seis variables climáticas obtenidas de WORLDCLIM (www. worldclim.org), (Bio1 = temperatura media anual, Bio5 = temperatura máxima del mes más caluroso, Bio6 = temperatura mínima de mes más frio, Bio12 = precipitación anual, Bio13 =precipitación de mes más húmedo, Bio14 = precipitación de mes más seco). Además de las variables climáticas, se obtuvo la elevación para cada uno de los registros a partir de un Modelo Digital de Elevación (MDE), y el tipo de vegetación asociado a cada uno de los registros obtenido a través del mapa de Vegetación y Uso de Suelo Serie IV del Instituto Nacional de Estadística, Geografía e Informática (INEGI 2009). 
Tabla 1. Localidades, ríos y cuencas con registros de nutria históricos*, y obtenidos en este estudio para Michoacán, México.

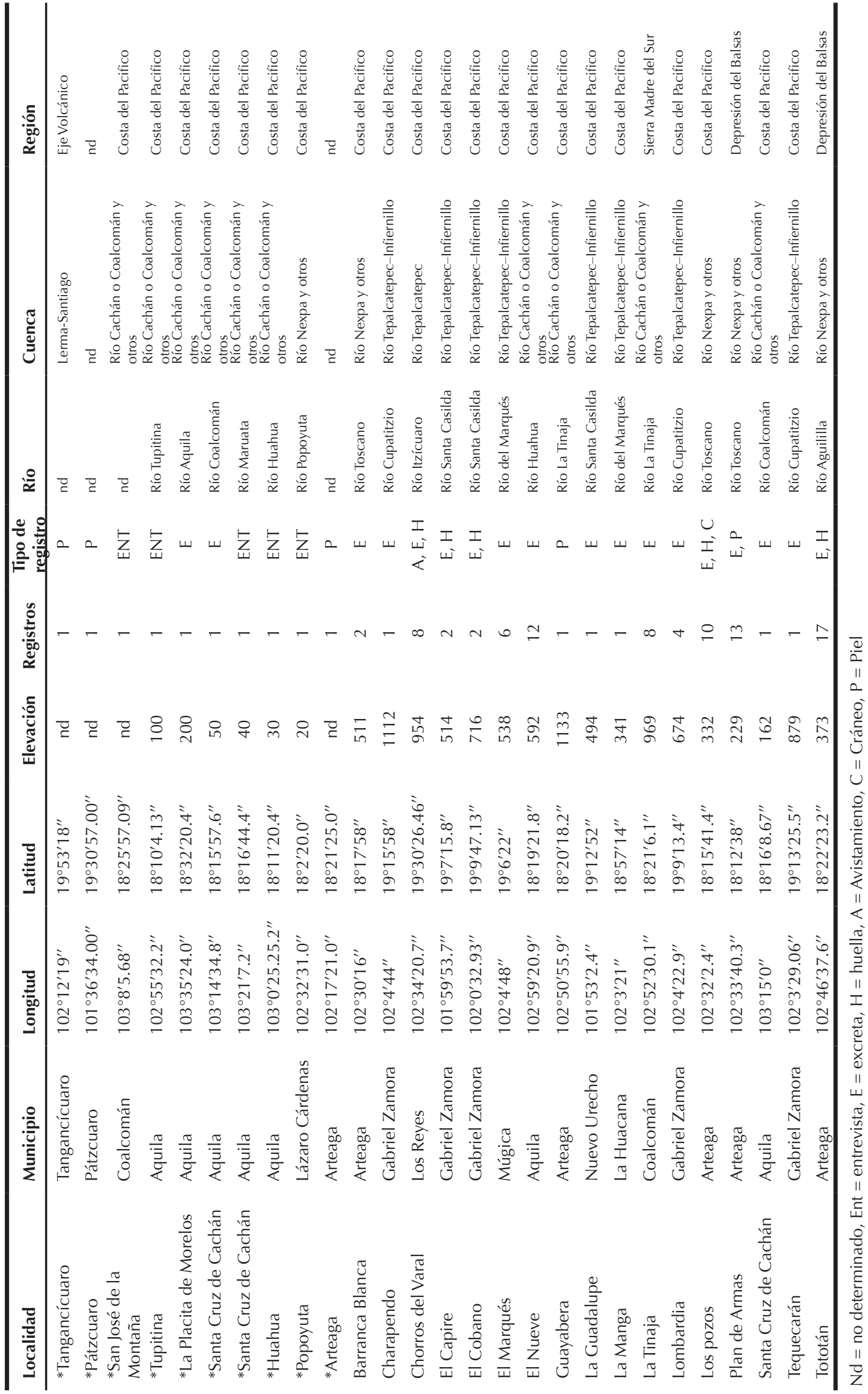


Revisión de literatura y base de datos. La presencia de la nutria de río en el estado de Michoacán se encontraba restringida a solamente 10 localidades de registro (Fig. 1 y Cuadro 1). La mayoría de los registros históricos existentes en bases de datos (6 de 10) corresponden a crónicas, relatos o entrevistas realizadas a pobladores (Brand 1961; Álvarez et al. 1987; Gallo 1997) por lo que carecían de datos precisos como localidad, vegetación o elevación, dos registros corresponden a rastros (Gallo 1997), y un registro corresponde a un ejemplar disecado y montado en la Colección del Laboratorio de Limnología de Pátzcuaro sin etiqueta de procedencia (Leopold 1959; Tabla 1).

vNueve de los 10 registros se encuentran georeferenciados en la base de datos de los mamíferos de Michoacán proporcionada por la Comisión Nacional para el Conocimiento y Uso de la Biodiversidad (CONABIO), a través del proyecto T009 "Actualización de la base de datos del Atlas Mastozoológico de México" (Ceballos 2002) y el proyecto P020 "Biodiversidad de los Mamíferos de Michoacán" (Álvarez-Solórzano y López-Vidal 1998).

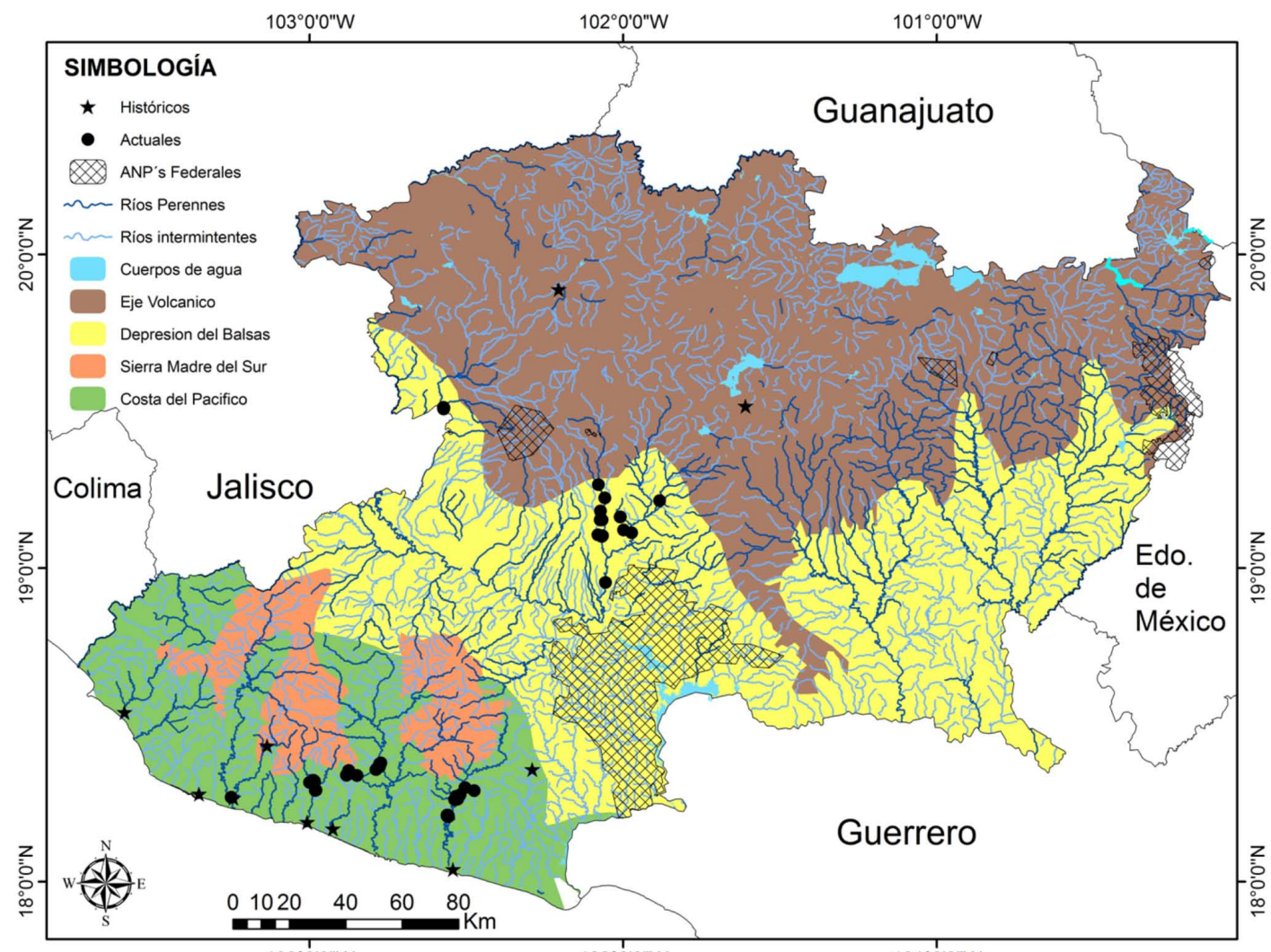

$103^{\circ} \mathrm{O}^{\prime} \mathrm{O}^{\prime \prime} \mathrm{W}$
Figura 1. Distribución de la nutria de río L. longicaudis en Michoacán, México.

Registros actuales. A partir de trabajo de campo se obtuvo 90 registros de 17 localidades (Tabla 1). Entre los 90 registros, 79 se lograron a partir de la colecta de excretas de letrinas ubicadas en su mayoría sobre rocas en medio de la corriente, seis de huellas, dos avistamientos, dos pieles, y un cráneo colectado y depositado en la colección de mastozoología de la Facultad de Biología de la Universidad Michoacana de San Nicolás de Hidalgos con el número de catálogo 3417 UMSNH (Fig. 2). 
Caracterización geográfica y ecológica de la nutria de río en Michoacán. Los 90 registros de campo obtenidos en este trabajo se encuentran localizados en ocho municipios del estado (Aquila, Coalcomán, Arteaga, La Huacana, Los Reyes, Mujica, Gabriel Zamora, Nuevo Urecho) a lo largo de tres grandes regiones biogeográficas del estado de Michoacán (CONABIO 1997). 61 registros en la región de la costa, 26 en la depresión del Balsas y tres en la Sierra Madre del Sur (Fig. 1). Los registros corresponden a cuatro cuencas hidrográficas (CNA 1998), la cuenca conocida como río Nexpa y afluentes (vertiente del Pacífico de la Sierra Madre del Sur y la Costa Michoacana) fue la que mayor cantidad de registros obtuvo (42, Tabla 1). En el río Toscano y el río Aguililla se observó la mayor cantidad de registros, 25 y 17 respectivamente. El resto de los registros se encuentran repartidos entre los ríos Huahua (13), la Tinaja (9), Itzícuaro (8), el Marqués (7), Cupatitzio (6), Santa Casilda (5) y Coalcomán (1) (Tabla 1). El intervalo de elevación en el que se registró a la especie fue entre 162 y 1,133 m.

Con base en la carta de vegetación y uso de suelo de INEGI (2009), el tipo de vegetación al cual se encuentran asociados los registros fueron el bosque tropical caducifolio ( $n$ $=47,52 \%)$, seguido del bosque de pino-encino $(n=14,29.7 \%)$ y bosque tropical subcaducifolio ( $n=13,14.4 \%$ ) pastizal cultivado ( $n=6,6.6 \%)$, y en menor número y porcentajes la agricultura de temporal (5), la agricultura de riego (4), el bosque de pino (2) y el bosque de encino con 1. Setenta y siete registros estaban asociados a vegetación natural y 68 de estos $(89.5 \%$ ) a vegetación conservada y continua en fragmentos $\geq a$ 1000 has.

La temperatura media anual asociada a los registros se encuentra entre los 21 y 28 ${ }^{\circ} \mathrm{C}$ mientras que el rango de temperaturas del mes más frio se encuentra entre los $10 \mathrm{y}$ $17^{\circ} \mathrm{C}$ y el rango de temperatura del mes más cálido se encuentra entre los 29 y $40{ }^{\circ} \mathrm{C}$. El rango de la precipitación media anual de los registros obtenidos se encuentra entre los 700 y $1300 \mathrm{~mm}$. Todos los registros se encuentran por debajo de los $300 \mathrm{~mm}$ de precipitación en el mes más lluvioso, y por debajo de $3 \mathrm{~mm}$ de precipitación en el mes más cálido.

\section{Discusión}

Los resultados reúnen el conjunto más abundante de registros recientes sobre $L$. longicaudis para el estado de Michoacán y quizás para cualquier otro estado del centro occidente de México. Hasta antes de este estudio, la presencia de la nutria en el estado de Michoacán se conocía de forma dispersa con registros obtenidos mediante entrevistas, rastros o ejemplares cazados sin especificar localidades o hábitats circundantes (Leopold 1959; Brand 1961; Álvarez et al. 1987; Gallo 1997, Magaña-Cota 2008). En este trabajo se presenta evidencia fotográfica y de ejemplares colectados de L. longicaudis (Fig. 2).

Para poder analizar todos los registros, se corrigió varios que provenían de la base datos de los mamíferos de Michoacán proporcionada por la CONABIO, que corresponden al proyecto T009 "Actualización de la base de datos del Atlas Mastozoológico de México"(Ceballos 2002). Se presentaron inconsistencias en localidad y municipio, un ejemplo es el registro de Brand (1961), el cual se ubica en el municipio de Coalcomán para la localidad de San José de la Montaña, sin embargo las coordenadas de la base de datos lo sitúan en el municipio de Angamacutiro en el altiplano, al límite con el estado de Guanajuato. Esta zona presenta poca probabilidad de presencia histórica 
de nutria. Recomendamos obtener la información de las fuentes originales, y consultar las coordenadas geográficas de localidades vecinas o cercanas para comparar y validar georeferenciación, evitando la distorsión de los registros a lo largo del tiempo y el uso e interpretación equivocada de los resultados. Una situación similar se documentó recientemente en el caso de la nutria de río para el estado de Durango (Charre-Medellín et al. 2011).

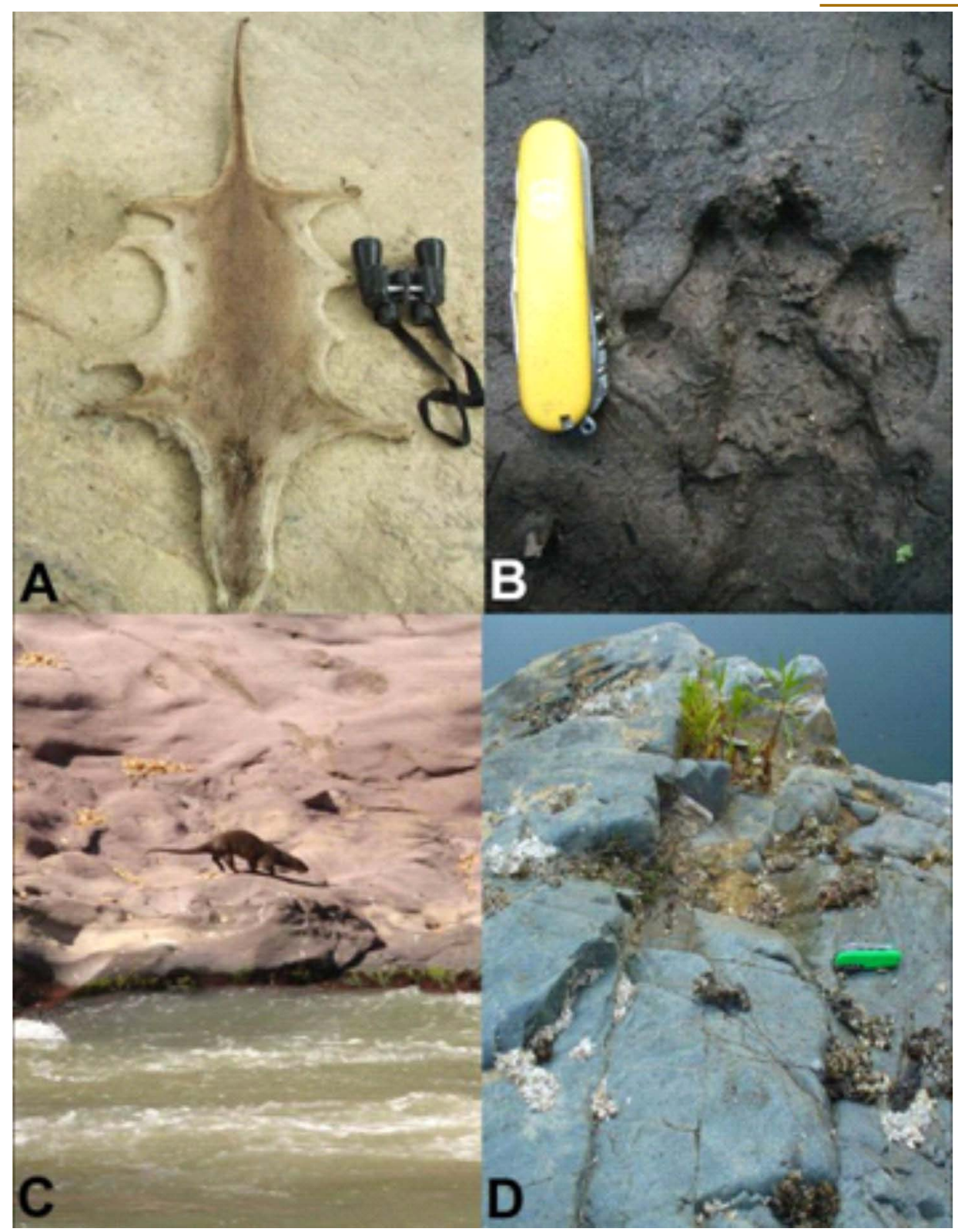

Figura 2. Evidencia de la presencia de nutria en el estado. A) Ejemplar cazado en la localidad de Plan de Armas, Municipio de Arteaga. B) Huellas de la localidad El Capire Municipio de Gabriel Zamora. C) Observación visual en la localidad de Chorros del Varal, Municipio de Los Reyes. D) Letrina en la localidad de Los Pozos, Municipio de Arteaga. Tablas

Con base en la distribución observada, la especie presenta una distribución actual en al menos tres regiones biogeográficas amplias de Michoacán; Balsas, Sierra Madre del Sur, y Costa del Pacífico. Sin embargo, es probable que en algunas partes del estado la nutria de río se distribuya localmente también en zonas bajas del Eje Neovolcánico, por ejemplo los registros de los ríos Cupatitzio e Itzícuaro, que corresponden respectivamente a los municipios de Gabriel Zamora y Los Reyes situados en los límites entre el Eje Neovolcánico y el Balsas (Tabla 1, Fig. 1). El intervalo de elevación registrado para la 
especie coincide de manera general con el amplio intervalo altitudinal descrito para la especie en general y para México (Larivière 1999; Santos-Moreno et al. 2003).

Con base en la revisión de literatura, la especie se encuentra asociada a bosques mesófilos, bosques tropicales perennifolios, subcaducifolios y caducifolios (Gallo y Casariego 2005). Pocos estudios han documentado la presencia de nutria asociada a bosques templados (Santos-Moreno et al. 2005), y en particular a bosques de pinoencino, el cual constituyó el segundo tipo de vegetación en Michoacán con mayor cantidad de registros $(n=14)$, solo después del bosque tropical caducifolio $(n=47)$, y similar al bosque tropical subcaducifolio $(n=13)$. La nutria de río es una especie que tolera modificaciones ambientales, ocupando áreas cercanas a zonas de actividad humana (Larivière 1999), por lo que no sorprende que se obtuvo registros en segmentos del río que cruzan zonas agropecuarias, a pesar de que las áreas agrícolas pueden presentar contaminación química u orgánica (Larivière 1999). La presencia de la nutria en sitios con efectos antropogénicos en Michoacán nos indica que a pesar de la presencia humana, los ríos cercanos a poblados parecen mantener un nivel de calidad ambiental dentro de los límites de tolerancia para la nutria, ya que se han reportado como muy sensibles a la contaminación de los cuerpos de agua (Soler 2002). Todos los registros obtenidos durante nuestro estudio están asociados a ríos con flujo perene, y en época seca varios forman pozas donde se facilita su observación (Fig. 1). La formación de pozas estacionales es común en los ríos del trópico de Michoacán durante la estación seca y crea microambientes que deben ser analizados en el futuro para determinar su influencia en la distribución de la nutria (Noviembre-Mayo). Si los ríos sufrieran disminución en sus cauces por canalizaciones, presas o sequía, existe el riesgo de que desaparecieran, afectando la disponibilidad de hábitat y posiblemente aislando poblaciones limitando su movilidad. Climáticamente todos los registros de campo se encontraron en sitios que durante el mes más seco (mayo) la precipitación es casi nula $(<3 \mathrm{~mm})$, y la temperatura sobrepasa los $30{ }^{\circ} \mathrm{C}$. Algunos pobladores estiman que durante la estación lluviosa, las nutrias se aventuran no solamente a los afluentes secundarios de los ríos perennes, sino también incursionan en arroyos ubicados a cientos de metros de los ríos principales, tal y como reporta Sánchez et al. (2007).

Michoacán puede constituir un área importante para la realización de estudios poblacionales de la especie, debido a su amplia red hidrológica y a su heterogeneidad ambiental a la cual observamos la presencia de la nutria de río, la cual va desde los bosques templados de la Sierra Madre del Sur y el Eje Neovolcánico, hasta los bosques tropicales caducifolios del las regiones del Balsas y la Costa. La heterogeneidad ambiental y separación de las cuencas ofrece un marco interesante para realizar estudios de genética de poblacionales de la especie, ya que es probable que existan potencialmente por lo menos dos poblaciones separadas por el parteaguas de la Sierra Madre del Sur, dividiendo la vertiente del pacifico y la vertiente interior que corresponde a la cuenca del bajo Balsas y el Tepalcatepec. Falta todavía determinar la amplitud de la distribución de la especie para cada región, hace falta investigación en los ríos ubicados en el norte de la costa de Michoacán (Municipio de Coahuayana), el norte de la Sierra Madre del Sur (Mpio. de Chinicuila), y al interior de las cuencas del río Tepalcatepec y Balsas (Mpio. de Tepalcatepec). También es importante documentar la presencia de la nutria en la reserva de la biosfera de Zicuirán-Infiernillo, principalmente en afluentes a la 
presa "Infiernillo" la cual parece tener un alto potencial de conectividad con el estado de Guerrero a través del río Balsas, y que pueden ser importantes para programas de conservación de la especie a nivel regional.

El presente estudio es resultado del trabajo de campo enfocado en mamíferos y aves en

Agradecimientos peligro de extinción en Michoacán, México. Agradecemos el apoyo en campo brindado por M. Álvarez-Jara, J. Venegas, A. Villanueva. Charre-Medellín agradece a CONACYT por la beca otorgada (239248). Este trabajo fue financiado por la Coordinación de Investigación Científica, Universidad Michoacana de San Nicolás de Hidalgo (UMSNH) y Fondos Mixtos CONACYT-Estado de Michoacán (Proyecto 41168). A la Facultad de Biología de la UMSNH por las facilidades otorgadas para la preparación de este manuscrito.

Álvarez, T., Arroyo-Cabrales, J., y M. González-Escamilla. 1987. Mamíferos (excepto Chiroptera) de la costa de Michoacán, México. Anales de la Escuela Nacional de Ciencias Biológicas, México 31:13-62.

Álvarez-Solórzano, T., y J. C. López-Vidal. 1998. Biodiversidad de los mamíferos en el Estado de Michoacán. México, D. F. Instituto Politécnico Nacional. Escuela Nacional de Ciencias Biológicas. Base de datos SNIB2010-CONABIO proyecto No. P020.

Bocco, G., M. Mendoza, A. Velázquez, y A. Torres. 1999. La regionalización geomorfológica como una alternativa de regionalización ecológica en México. El caso de Michoacán de Ocampo. Investigaciones Geográficas 40:7-21.

Brand, D. 1961. Coalcomán and Motines del Oro. An ex-district of Michoacán, México. The Hague: Martinus Nijhoff, Texas, EUA, University of Texas. Austin, EE.UU.

Ceballos, G. 2002. Actualización de la base de datos del Atlas Mastozoológico de México. Universidad Nacional Autónoma de México. Instituto de Ecología. Bases de datos SNIB2010-CONABIO proyectos No. T009 y A003. Ciudad de México, México.

Ceballos, G., y G. Oliva. 2005. Los mamíferos silvestres de México. CONABIO - Fondo de Cultura Económica. Ciudad de México, México.

Charre-Medellín, J. F. 2009. Diversidad y distribución de mamíferos medianos y grandes en el municipio de Arteaga, Michoacán. Tesis de licenciatura. Facultad de Biología, Universidad Michoacana de San Nicolás de Hidalgo. Morelia, Michoacán, México.

Charre-Medellín, J. F., C. López-González, A. Lozano, y A. F. Guzmán. 2011. Conocimiento actual sobre la nutria neotropical (Lontra longicaudis annectens) en el estado de Durango, México. Revista Mexicana de Biodiversidad 82:1343-1347.

CONABio (Comisión Nacional para el Conocimiento y Uso de la Biodiversidad). 1997. Provincias biogeográficas de México. Escala 1:4 000 000. Comisión Nacional para el Conocimiento y Uso de la Biodiversidad. Ciudad de México, México.

CNA (Comisión Nacional del Agua). 1998. Cuencas Hidrológicas. Escala 1:250000. México. 
EisenberG, J. F., y K. H. Redford. 1999. Mammals of the Neotropics. Vol. 3. The central Neotropics: Ecuador, Perú, Bolivia, Brazil. The University of Chicago Press. Chicago, EE.UU.

Gallo, J. P. 1997. Situación y distribución de las nutrias en México, con énfasis en Lontra longicaudis annectens, Major, 1897. Revista Mexicana de Mastozoología 2:10-32.

Gallo, J. P., y M. A. Casariego. 2005. Lontra longicaudis (Olfers, 1818). Pp. 374-376 en Los mamíferos silvestres de México (Ceballos, G., y G. Oliva, eds.). CONABIOFondo de Cultura Económica. Ciudad de México, México.

Gallo, J. P. 2008. Los perros de agua, perspectivas históricas. Conferencia magistral, IX Congreso Nacional de Mastozoología, 22 a 26 de septiembre de 2008. Autlán de La Grana, Jalisco, México.

Guerrero-Flores, J. J., S. Macías-Sánchez, V. Mundo-Hernández, y F. Méndez-Sánchez. 2013. Ecología de la nutria (Lontra longicaudis) en el municipio de Temascaltepec, estado de México: estudio de caso. Therya 4:231-242.

INEGi (Instituto Nacional de Estadística y Geografía). 2009. Carta de Uso del Suelo y Vegetación, Serie IV, escala 1:250000. Ciudad de México, México.

Israde-Alcántara, I. 2005. Los cuerpos de agua. Pp. 32-37 en La Biodiversidad en Michoacán. Estudio de estado. Morelia, Michoacán, México (Villaseñor-Gómez, L. E., y O. A. Leal-Nares, eds.). Comisión Nacional para el conocimiento y uso de la Biodiversidad, Gobierno del estado de Michoacán, Secretaría de Urbanismo y Medio Ambiente y Universidad Michoacana de San Nicolás de Hidalgo. Morelia, México.

LARIVIÈre, S. 1999. Lontra longicaudis. Mammalian Species 609:1-5.

Leopold, A. S. 1959. Fauna silvestre de México. Instituto Mexicano de Recursos Naturales Renovables. Ciudad de México, México.

López Witchis, R. 1998. Base de datos de mamíferos de México depositados en colecciones de Estados Unidos y Canadá. Universidad Autónoma Metropolitana. Unidad Iztapalapa. Bases de datos SNIB-CONABIO. Proyecto No. P130. Ciudad de México, México.

Magaña-Cota, G. 2008. Notas de zoología tomadas durante mi estancia en México. Cuaderno 2. Alfredo Dugès. Universidad de Guanajuato. Guanajuato, México.

Quadros, J., y L. A. Monteiro-Filho. 2000. Fruit occurrence in the diet of the Neotropical Otter, Lontra longicaudis in southern Brazilian Atlantic forest and its implication for seed dispersion. Mastozoología Neotropical 7:33-36.

Reid, F. 1997. A field Guide to the Mammals of Central America and southeast México. Oxford University Press. Oxford, EE.UU.

Sánchez, O., R. Medellín, A. Aldama, B. Goettsch, J. Soberón, y M. Tambutti. 2007. Método de evaluación del riesgo de extinción de las especies silvestres en México (MER). Instituto Nacional de Ecología (INE-SEMARNAT). Ciudad de México, México.

Santos-Moreno, J., M. Briones-Salas, G. González-Pérez, y T. Ortiz. 2003. Networthy records of two rare mammals in Sierra Norte de Oaxaca, México. The Southwestern Naturalist 48:312-313.

Soler, A. 2002. Nutrias por todo México. Biodiversitas 43:13-14. 
Sometido: 11 de septiembre de 2013

Revisado: 30 de noviembre de 2013

Aceptado: 10 de enero de 2014

Editor asociado: Juan Pablo Gallo

Diseño gráfico editorial: Gerardo Hernández 